\title{
Os odontoideum. Presentación en edad adulta
}

\author{
Os odontoideum. Presentation in adult age \\ García-Ramos CL, ${ }^{*}$ Mireles-Cano JN, ${ }^{\ddagger}$ Rosales-Olivarez LM, ${ }^{\S}$ Alpizar-Aguirre A, * Reyes-Sánchez A \\ Instituto Nacional de Rehabilitación, Ciudad de México.
}

RESUMEN. Os odontoideum es un osículo oval o redondo de tamaño variable con un borde cortical liso, que se corresponde parcialmente con la apófisis odontoides, sin tener continuidad ósea con el resto de C2. La etiología es multifactorial, causa inestabilidad y clínicamente se traduce en dolor y datos de compresión a las estructuras neurales. El tratamiento de elección es quirúrgico y se han desarrollado técnicas que se enfocan en conservar la estabilidad del segmento. Presentamos el caso de una mujer de 23 años, inicia padecimiento a los ocho años, refiere cervicalgia moderada a intensa, que evoluciona con parestesias en hemicuerpo izquierdo y posteriormente paresia de miembro torácico izquierdo. A la exploración física se evidencia hipoestesia de hemicuerpo izquierdo, así como paresia de miembro torácico izquierdo. Los estudios de extensión demuestran lesión axonal crónica de C1 a C3, de predominio izquierdo, los estudios de imagen evidencian inestabilidad axial y la resonancia magnética compresión bulbar. La paciente recibe tratamiento quirúrgico consistente en fijación posterior $\mathrm{C} 1$ $\mathrm{C} 2$, evolucionando satisfactoriamente.

Palabras clave: Os odontoideum, tratamiento quirúrgico, fijación interna, tornillos pediculares, fusión de columna.

\section{Introducción}

El atlas y la apófisis odontoides se originan del primer esclerótomo cervical con algunas contribuciones de remanentes del proatlas, mientras que el cuerpo, masas laterales y arco posterior del axis provienen por completo del segundo esclerótomo cervical.
ABSTRACT. The os odontoideum is a variable oval or round ossicle with a smooth cortical border, which partially corresponds to the odontoid process, without having continuity with the rest of C2 bone. The multifactorial etiology causes instability and clinically translates into pain and compression data into neural structures. The treatment of choice is surgical and techniques have been developed that focus on preserving the stability of the segment. We present the case of a 23-year-old female patient, who begins to suffer at 8 years of age, refers to moderate to severe cervicalgia, which develops with paresthesias in the left hemisphere and later paresis of the left thoracic limb. Physical examination showed hypoaesthesia of the left hemisphere, as well as paresis of the left thoracic limb. Extension studies demonstrate chronic axonal lesion from C1 to C3, predominantly left, imaging studies showing axial instability and magnetic resonance bulbar compression. The patient receives surgical treatment consisting of posterior fixation $\mathrm{C} 1-\mathrm{C} 2$, evolving satisfactorily.

Keywords: Os odontoideum, surgical treatment, internal fixation, pedicle screw, spinal fusion.

La apófisis odontoides se separa entre la sexta y séptima semana de gestación, moviéndose caudalmente para unirse al cuerpo del axis. ${ }^{2,3}$ La unión craneocervical es uno de los sitios más comunes para malformaciones. ${ }^{1}$

El os odontoideum es un osículo oval o redondo de tamaño variable con un borde cortical liso, que se corresponde de manera parcial con la apófisis odontoides, sin tener

\footnotetext{
* Médico adscrito al Servicio Cirugía de Columna Vertebral, Instituto Nacional de Rehabilitación, México.

‡ Neurocirujano, Cirugía de Columna Vertebral. Hospital Regional de Alta Especialidad del Bajío, León, Guanajuato, México.

$\S$ Jefe del Servicio Cirugía de Columna Vertebral, Instituto Nacional de Rehabilitación, México.

" Jefe de División de Cirugía de Columna, Instituto Nacional de Rehabilitación, México.
}

\section{Dirección para correspondencia: \\ Dr. Alejandro Reyes-Sánchez \\ Camino a Santa Teresa Núm. 1055-950, \\ Col. Héroes de Padierna, CP 10700, Ciudad de México, México. \\ Tel. 5999-1000, ext. 12206, \\ E-mail: alereyes@inr.gob.mx}

Citar como: García-Ramos CL, Mireles-Cano JN, Rosales-Olivarez LM, Alpizar-Aguirre A, Reyes-Sánchez A. Os odontoideum. Presentación en edad adulta. Acta Ortop Mex. 2020; 34(4): 234-237. https://dx.doi.org/10.35366/97557

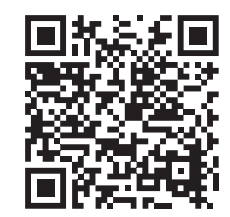


continuidad ósea con el resto de C2, éste puede localizarse en posición normal de la apófisis odontoides (ortotópico) o cerca de la base del hueso occipital en el área del foramen magno (diatópico), donde puede fusionarse con el clivus. ${ }^{4,5}$

La etiología del os odontoideum ha sido atribuida a diversas causas como embriológica, traumática o vascular, ${ }^{5}$ también se suele asociar a insuficiencia ligamentaria e inestabilidad C1-C2. ${ }^{6}$

Se manifiesta radiológicamente con inestabilidad anteroposterior de C1-C2, con un aumento del espacio entre el arco anterior del atlas y la apófisis odontoides, medida que no debe superar los $3 \mathrm{~mm}$ en adultos; ${ }^{7}$ una movilidad de la odontoides mayor de $6 \mathrm{~mm}$ en relación con el arco anterior del atlas suele implicar la rotura del ligamento transverso, con aumento de riesgos neurológicos.

En el ámbito clínico, la inestabilidad aguda puede acompañarse de afección neurológica e incluso con muerte súbita, la inestabilidad crónica se presenta con dolor en la nuca y aparición progresiva de datos neurológicos de compresión bulbar.
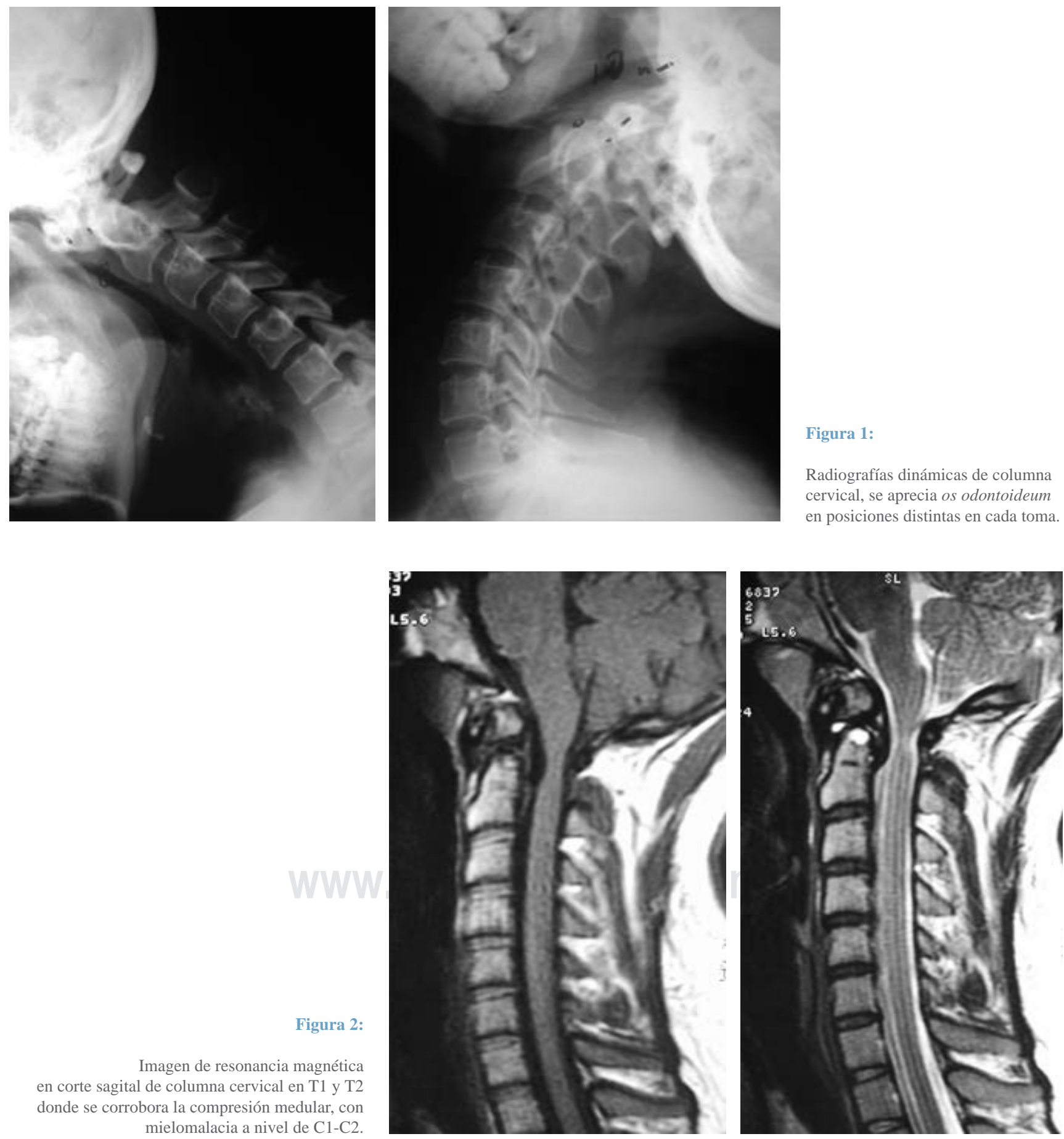

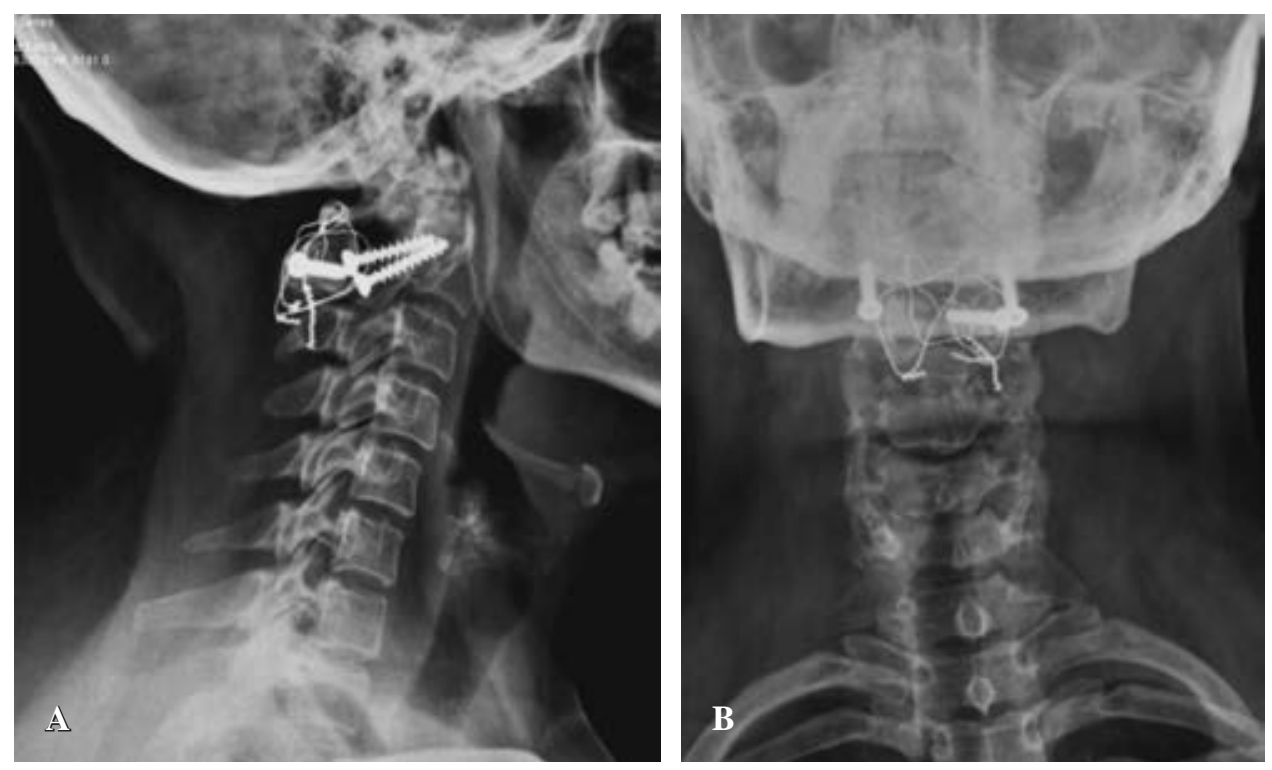

Figura 3:

Radiografías postoperatorias lateral (A) y anteroposterior de columna cervical (B). Se observa la fijación posterior de C1-C2 con técnica de Magerl.

El tratamiento de elección es quirúrgico, en especial cuando se desarrolla daño neurológico progresivo o los estudios radiológicos muestran inestabilidad dinámica, los procedimientos quirúrgicos empleados pretenden la restauración del conducto medular, la artrodesis ósea C1-C2 posterior es el resultado más favorable a largo plazo. ${ }^{8}$ Se han desarrollado diversas técnicas de fijación para conseguir estos objetivos: la técnica de Gallie, con alambres sublaminares en $\mathrm{C} 1$ a apófisis espinosa de $\mathrm{C} 2$; la técnica de Brooks, con alambres sublaminares bilaterales en C1-C2; la técnica de Magerl con tornillos transarticulares C1-C2 o con ganchos laminares de Halifax. ${ }^{9}$

\section{Caso clínico}

Reporte de caso clínico de una mujer de 23 años, quien niega antecedentes de importancia, incluidos traumáticos, inició a los ocho años con dolor cervical posterior de moderada intensidad, que incrementó su intensidad de manera progresiva, a los 18 años nota parestesias en hemicuerpo izquierdo y a los 20 años nota paresia de miembro torácico izquierdo, a la exploración física se evidencia hipoestesia de hemicuerpo izquierdo, así como paresia de miembro torácico izquierdo, la electromiografía reporta datos de lesión axonal crónica de C1 a C3, de predominio izquierdo.

En las radiografías dinámicas de columna cervical se observa un espacio interdental anterior de $8 \mathrm{~mm}$ y un espacio interdental posterior de $10 \mathrm{~mm}$, así como la presencia de os odontoideum (Figura 1). Con la resonancia magnética de unión craneocervical se identifica compresión de la unión bulbomedular, con imagen hiperintensa medular en secuencia T1 a nivel C1 (Figura 2).

Se realiza fijación posterior mediante colocación de alambre sublaminar C1-C2, colocación de tornillos transarticulares C1-C2 e injerto autólogo de cresta ilíaca (Figura 3).

\section{Resultados}

La paciente evolucionó de manera satisfactoria con fuerza global 5/5 y presentó mejoría de la sensibilidad de hemicuerpo izquierdo (2/2).

Los estudios radiológicos postoperatorios mostraron una reducción de la luxación y la restauración del conducto medular cervical, sin datos de fatiga ni aflojamiento del material al seguimiento a los 24 meses, así como consolidación adecuada del injerto.

\section{Discusión}

Desde la primera descripción de os odontoideum hecha por Giacomini en 1886, se han planteado diversas etiologías que aún permanecen sin dilucidarse; se ha propuesto la teoría postraumática por Fielding y colaboradores, que hipotetiza la presencia de una fractura no reconocida del diente seguida de una contracción del ligamento alar, lo que conduce a distracción del fragmento con interrupción del riesgo sanguíneo y a la formación de un osículo; ${ }^{4}$ otra teoría es la congénita, Wollin describió una incompleta osificación a través del disco intervertebral vestigial, separando el apófisis odontoides del cuerpo del axis, lo cual forma un osículo; ${ }^{10}$ y por último, la etiología genética sugerida al demostrarse cambios óseos y ligamentarios en un estudio de necropsia en gemelos. ${ }^{5}$

\section{Conclusiones}

El os odontoideum es una patología rara que se presenta y se diagnostica de manera predominante en la etapa infantil.

La etiología es controversial, pero la mayoría de los autores sugieren etiología traumática. En el ámbito clínico, se manifiesta con dolor cervical y suele asociarse con signos y síntomas neurológicos de compresión de la unión bulbomedular. 
Bibliografía

1. VanGilder JC, Menezes AH, Dolan KD. Embryology and development of the craniovertebral junction. In: VanGilder JC, Menezes AH, Dolan KD. The craneovertebral junction and its abnormalities. Mount Kisco, NY: Futura, 1988, 1-8.

2. Hadley MN. Os odontoideum. Neurosurgery. 2002; 50(3): S148-S155.

3. O’Rahilly R, Meyer DB. The timing and sequence in the development of the human vertebral column during the embryonic period proper. Anat Embryol. 1979; 157: 167-176.

4. Fielding JW, Hensinger RN, Hawkins RJ. Os odontoideum. J Bone Joint Surg Am. 1980; 62: 376-83.

5. Dai L, Yuan W, Ni B, et al. Os odontoideum: etiology, diagnosis, and managment. Surg Neurol. 2000; 53: 106-8.

6. Martínez-Flores P, Chillón-Medina D, Escosa-Bage M, ManzanaresSoler R. Subluxación atloaxoidea. "Os odontoideum”. Reducción y fijación posterior con alambres sublaminares en C1 anclados en tornillos pediculares de C2. Caso clínico. Neurocirugía. 2004; 15: 604-8.

7. Stillerman CB, Roy RS, Weiss MH. Cervical spine injuries: diagnosis and managment. En: Rengachary W (ed). Neurosurgery. Cap. 285, 2nd ed. New York: McGraw-Hill, 1986, 2875-904.

8. Dickman CA, Sonntag VKM, Papadopoulus SM, Hadley MN: The interspinous method of posterior atlantoaxial arthrodesis. J Neurosurg. 1991; 74: 190-8.

9. Grob D, Crisco JJ, Panjabi MM, Wang P, Dvorak J. Biomechanical evaluation of four different posterior atlantoaxial fixation techniques. Spine. 1992; 17: 480-9.

10. Wollin DG. The os odontoideum: separate odontoid process. J Bone Joint Surg Am. 1963; 45: 1459-71.

Conflicto de intereses: En el presente estudio, no existe conflicto de intereses de ninguno de los autores para su realización. 